\title{
Pseudo-Supplemented Almost Distributive Fuzzy Lattices
}

\author{
Gerima Tefera Dejen \\ Department of Mathematics, College of Natural Science, Wollo University, Dessie, Ethiopia.
}

\begin{abstract}
How to cite this paper: Gerima Tefera Dejen. (2021) Pseudo-Supplemented Almost Distributive Fuzzy Lattices. Journal of Applied Mathematics and Computation, 5(2), 119-123.

DOI: $10.26855 /$ jamc.2021.06.006
\end{abstract}

Received: April 6, 2021

Accepted: April 30, 2021

Published: May 27, 2021

*Corresponding author: Gerima Tefera Dejen, Department of Mathematics, College of Natural Science, Wollo University, Dessie, Ethiopia.

Email: gerima233@gmail.com

\begin{abstract}
The introduction of an almost distributive lattice as a common abstraction of the existing lattice and ring-theoretic generalization of a Boolean algebra lead to the development of many other related theories. In addition, the concepts of almost distributive fuzzy lattice as a new theory are introduced. As a result, the concepts of Birkhoff center in almost distributive fuzzy lattice, B-almost distributive fuzzy lattice, and others was introduced. In this paper, the concept of Pseudo-Supplemented Almost Distributive Fuzzy Lattice is introduced. Birkhoff center in Pseudo-Supplemeted Almost Distributive Fuzzy Lattice is explained. The extension of Pseudo-Supplemented Almost Distributive Lattice to Pseudo-Supplemented Almost Distributive Fuzzy Lattice is investigated. The notion of Relatively Complemented Almost Distributive Fuzzy Lattice is explained; $(R, A)$ is Pseudo-Supplemented Almost Distributive Fuzzy Lattice if and only if $\mathrm{R}$ is Pseudo-Supplemented Almost Distributive Lattice. $(R, A)$ is a pseudo-complemented ADFL with Birkhoff center $B_{A}(R)$ if and only if $\mathrm{R}$ is a pseudo-complemented almost distributive lattice with Birkhoff center B.
\end{abstract}

\section{Keywords}

Almost Distributive Lattice, Almost Distributive Fuzzy Lattice, Pseudo-Supplemented Almost Distributive Lattice, Pseudo-Supplemented Almost Distributive Fuzzy Lattice, Pseudo-Complemented Almost Distributive Lattice

\section{Introduction}

The concept of an almost distributive lattice (ADL) as a common abstraction of the existing lattice and ring-theoretic generalization of a Boolean algebra introduced by Swamy and Rao [1], and Roa and Mihret [2] investigated basic ideas on properties of stone almost distributive fuzzy lattice. The class of ADL'S with pseudo-complementation was introduced by Swamy, Rao and Nanaji [3] and it was observed that an ADL can have more than one pseudo-complementation. The notion of pseudo-supplemented almost distributive lattice (PSADL) as a generalization of pseudo-supplemented lattice was introduced by Rao and Naveen [4]. The set PI(R) of all principal ideal of R is a distributive lattice under the operations defined and which is the least element.

Zadeh first introduced the concept of fuzzy sets [5], and Chon defined a fuzzy lattice as a fuzzy relation and characterized it [6]. The concept of almost distributive fuzzy lattice (ADFL) was introduced by Berhanu A., Yohannes G. andBekalu T. [7] as a generalization of distributive fuzzy lattice (DFL) and the idea of Birkhoff center of almost distributive fuzzy lattice initiated by Berhanu A. and Gerima T. [8], and Berhanu and et al. [9] introduced B-Almost Distributive Fuzzy lattice.

In this paper, we introduce the notion of pseudo- supplemented almost distributive fuzzy lattice (PSADFL) as a gene- 
ralization of pseudo-supplemented distributive fuzzy lattice. Throughout this paper, $(R, A)$ and $B_{A}$ represente an ADFL and Birkhoff center of an almost distributive fuzzy lattice $(R, A)$ with fuzzy subset A respectively.

\section{Methods}

Definition 2.1. [1] An algebra $(R, \vee, \wedge, 0)$ of type $(2,2,0)$ is called an almost distributive lattice (ADL) if the following condition holds:

1. $a \wedge(b \vee c)=(a \wedge b) \vee(a \wedge c)$.

2. $(a \vee b) \wedge c=(a \wedge c) \vee(b \wedge c)$.

3. $(a \vee b) \wedge a=a$.

4. $(a \vee b) \wedge b=b$.

5. $a \vee(a \wedge b)=a$.

6. $0 \wedge a=0$.

Lemma 2.2. [1] Let $(R, \vee, \wedge, 0)$ be an ADL. For any $a, b, c \in R$ we have the following:

1. $a \vee b=a \Leftrightarrow a \wedge b=b$.

2. $a \vee b=b \Leftrightarrow a \wedge b=a$.

3. $\wedge$ is associative.

4. $a \wedge b \wedge c=b \wedge a \wedge c$.

5. $a \wedge b=0 \Leftrightarrow b \wedge a=0$.

Definition 2.3. [1] Let R be an ADL with 0. For any $a, b \in R$, define $a \leq b$ if and only if $a \wedge b=a$ or equivalently $a \vee b=b$. Then " $\leq "$ is a partial ordering on $\mathrm{R}$.

Lemma 2.4. [1] Let $\mathrm{R}$ be an ADL with zero, and $m \in R$. Thenthe following are equivalent:

1. $m$ is maximal with respect to the partial ordering $\leq$.

2. $m \vee a=m$, for all $a \in A$.

3. $m \wedge a=a$, for all $a \in R$.

Definition 2.5.[3] Let $(\mathrm{R}, \vee, \wedge, 0)$ be an ADL with 0 . Then a unitary operation $a \rightarrow a^{*}$ on $\mathrm{R}$ is called a pseudo- complementation on $\mathrm{R}$ if the following hold for all $a, b \in R$ :

1. $a \wedge a^{*}=0$.

2. $a \wedge b=0 \Rightarrow a^{*} \wedge b=b$.

3. $(a \vee b)^{*}=a^{*} \wedge b^{*}$, $R$.

Here the unary operation $*$ is called a pseudo- complementation on $R$ and $a^{*}$ is called a pseudo- complement of a in

Definition 2.6. [4] Let $\mathrm{R}$ be an ADL with a maximal element $\mathrm{m}$ and Birkhoff center $B$. Then $\mathrm{A}$ is called a pseudo-supplemented almost distributive lattice (PSADL) if each $x \in R$, there exists $b \in B$ such that

$P_{1}: x \wedge b=b$.

$P_{2}:$ If $c \in B$ and $x \wedge c=c$, then $b \wedge c=c$.

In this case $b \wedge m$ is uniquely determined by $x$ and it is denoted by $x$ !.

Thus $x ! \in B([0, m])$. We call $x$ ! is a pseudo- supplement of $x$.

Definition 2.7 [6] Let $\mathrm{R}$ be a non-empty set. Then a function $A: R \times R \rightarrow[0,1]$ is called a fuzzy relation in $R$. The fuzzy relation $A$ in $R$ is reflexive if and only if $A(a, a)=1$, for all $a \in R$, $A$ is antisymmetric if and only if $A(a, b)+A(b, a)>1$ implies $a=b$. or $A(a, b)=A(b, a)>0$ implies $a=b$ and $A$ is transitive if and only if $A(a, c) \geq \sup _{b \in R} \min (A(a, b), A(b, c))>0$.

Let $(R, A)$ be a fuzzy partially ordered set. Then $(R, A)$ is a fuzzy lattice if and only if $a \vee b$ and $a \wedge b$ exist for all $a, b \in R$. $(R, A)$ be a fuzzy lattice $(R, A)$ is distributive if and only if $a \wedge(b \vee c)=(a \wedge b) \vee(a \wedge c) \quad$ and $(a \vee b) \wedge(a \vee c)=a \vee(b \wedge c)$, for all $a, b, c \in R$. 
Definition 2.8. [7] Let $(R, \vee, \wedge, 0)$ be an algebra of type $(2,2,0)$ and $(R, A)$ be a fuzzy poset. Then $(R, A)$ is an almost distributive fuzzy lattice (ADFL) if the following hold for all $a, b, c \in R$ :

1. $A(a \vee 0, a)>0$.

2. $A(0 \wedge a, 0)>0$.

3. $A((a \vee b) \wedge c,(a \wedge c) \vee(b \wedge c))=1$.

4. $A(a \wedge(b \vee c),(a \wedge b) \vee(a \wedge c))=1$.

5. $A(a \vee(b \wedge c),(a \vee b) \wedge(a \vee c))=1$.

6. $A(b,(a \vee b) \wedge b)>0$.

An ADFL $(R, A)$ is said to be relatively complemented ADFL if $([a, b], A)$ is a complemented fuzzy lattice for any $a, b \in R$ with $A(a, b)>0$. Let $R$ be a non-empty set. Then $\mu: R \rightarrow[0,1]$ is called a fuzzy subset of $R$.

\section{Results}

\subsection{Pseudo-Supplemented Almost Distributive Fuzzy Lattice (PSADFL).}

Definition3.1. Let $\mathrm{R}$ be an ADFL with maximal element $\mathrm{m}$ and

$B_{A}=\{a \in R \mid A(a \wedge b, 0)>0$ and $A(x,(a \vee b) \wedge x)>0\}$ for some $b, x \in R$. Then $\left(B_{A}, \vee, \wedge\right)$ is a relatively complemented almost distributive fuzzy lattice. It is called Birkhoff center of an almost distributive fuzzy lattice.

It can be observed that for any $b \in B_{A}, b \wedge m$ is complemented element in the distributive fuzzy lattice $[0, m]_{A}$ with $A\left((b \wedge m) \wedge(b \wedge m)^{\prime}, 0>0\right.$ and $A\left(m,(b \wedge m) \vee(b \wedge m)^{\prime}\right)>0$.

Theorem 3.2. For any $a \in R, b \in B_{A}$ if and only if $a \wedge x \in B_{A}([0, x])$ for each $x \in R$.

Proof. Let $a \in B_{A}$ and $x \in R$. Then there exists $c \in R$ such that $A(0, a \wedge c)>0$ and $A(x,(a \vee c) \wedge x)>0$.

Now, $A((a \wedge x) \wedge(c \wedge x), 0)=A((a \wedge c) \wedge x \wedge x, 0)$

$=A(a \wedge c \wedge x, 0)=A(0 \wedge x, 0)=A(0,0)=1>0$.

and $A((a \wedge x) \vee(c \wedge x), x)=A(((a \wedge x) \vee c) \wedge(a \wedge x) \vee x, x)$

$=A(((a \wedge x) \vee c) \wedge x, x)=A((c \vee(a \wedge x)) \wedge x, x)=A((c \vee a) \wedge(c \vee x)) \wedge x, x)$

$=A((c \vee x) \wedge x, x)=A(x, x)=1>0$.

Hence $A((a \wedge x) \wedge(c \wedge x), x)>0$ and $A((a \wedge x) \vee(c \wedge x), x)>0$.

Thus $a \wedge x \in B_{A}([0, x])$.

Conversely, suppose $a \in R$ and for each $x \in R, a \wedge x \in B_{A}([0, x])$. Let b be the complement of $a \wedge m \in B_{A}([0, m])$.

Then $A(0, a \wedge m \wedge b)=A(0,0)=1>0$ and $A(m,(a \wedge m) \vee b)=A(m, m)=1>0$, since $b \in[0, m]$.

Thus $a \in B_{A}$.

Theorem 3.3. For any $a \in B_{A}, B_{A}([0, a]) \subseteq B_{A}$ and hence $B_{A}([0, a])=\left\{b \wedge a \mid b \in B_{A}\right\}$.

Proof. Let $c \in B_{A}([0, a])$. Then there exists $d \in[0, a]$ such that $A(0, c \wedge d)>0$ and $A(a, c \vee d)>0$. Since $a \in B_{A}$ there exists $b \in R$ such that $A(0, a \wedge b)>0$ and $A(x,(a \vee b) \wedge x)>0$.

Now $A(c \wedge(d \vee b), 0)=A((c \wedge d) \vee(c \wedge b), 0)$.

$=A(0 \vee(c \wedge b), 0)=A(c \wedge b, 0)=A(c \wedge a \wedge b, 0)=A(c \wedge 0,0)=A(0,0)=1>0$.

and $A(a \vee b,[c \vee(d \vee b)] \wedge(a \vee b))=A((a \vee b,[(c \vee d) \vee b] \wedge(a \vee b)$

$=A(a \vee b,(a \vee b) \wedge(a \vee b))=A(a \vee b, a \vee b)=1>0$

Hence $A(c \wedge(d \vee b), 0)>0$ and $A(a \vee b,[c \vee(d \vee b)] \wedge(a \vee b))>0$.

Hence $c \vee(d \vee b)$ is maximal element in R.

Thus $c \in B_{A}$ and by theorem $3.2 c \wedge a \in B_{A}([0, a])$ for all $c \in B_{A}$.

Theorem 3.4. $B_{A}(P I(R))=\left\{(b]_{A} \mid b \in B_{A}\right\}$.

Proof. Suppose $(b]_{A} \subseteq B_{A}(P I(R))$ for some $b \in R$. Then there exists $(c] \in P I(R)$ 
such that $(b]_{A} \wedge(c]_{A}=(b \wedge c]_{A}=(0]_{A}$ and $(b]_{A} \vee(c]_{A}=(b \vee c]_{A}=R$.

Thus $b \in B_{A}(R)$.

Conversely, suppose $b \in B_{A}(R)$. Then there exists an element $c \in R$ such that $A(0, b \wedge c)>0$ and $A(x,(b \vee c) \wedge x)>0$.

So that $(b]_{A} \wedge(c]_{A}=(b \wedge c]_{A}=(0]_{A}$ and $(b]_{A} \vee(c]_{A}=(b \vee c]_{A}=R$.

Hence $(b]_{A} \subseteq B_{A}(P I(R))$.

Theorem 3.5. If $b, c \in R$ such that $(c]_{A}=(b]_{A}$, then $b \in B_{A}(R)$ if and only if $c \in B_{A}(R)$.

Definition 3.6. Let $\mathrm{R}$ be a distributive fuzzy lattice with 0,1 and $B_{A}(R)$ be its Birkhoff center of $R$. If for any $x \in R$, there exists a greatest element $b \in B_{A}(R)$ such that $A(b, x)>0$, then $R$ is called a Pseudo-Supplemented in almost distributive fuzzy lattice.

Definition 3.7. Let $(R, A)$ be an ADFL with a maximal element $m$ and Birkhoff center $B_{A}(R)$. Then $(R, A)$ is called a Pseudo-Supplemented Almost Distributive Fuzzy Lattice (PSADFL) if for each $x \in R$, there exists $b \in B_{A}(R)$ such that

$P_{1}: A(b, x \wedge b)>0$.

$P_{2}$ : If $c \in B_{A}(R)$ and $A(c, x \wedge c)>0$, then $A(c, b \wedge c)>0$.

In this case $b \wedge m$ is uniquely determined by $x$ and denoted by $x$ !

Thus $x ! \in B_{A}([0 . m])$ by Theorem 3.2.We call $x$ ! a pseudo complement of $\mathrm{x}$.

Theorem 3.8. $(R, A)$ is a Pseudo-Supplemented Almost Distributive Fuzzy Lattice(PSADFL) with maximal element if and only if $R$ is a Pseudo-Supplemented Almost Distributive Lattice (PSADL) with maximal element.

Theorem 3.9. $(R, A)$ is a pseudo- complemented ADFL with Birkhoff center $B_{A}(R)$ if and only if $\mathrm{R}$ is a pseudocomplemented ADL with Birkhoff center $B$.

Remark 3.10. If $(R, A)$ is a PSADFL, $c \in B_{A}(R)$ and $A(c, x \wedge c)>0$, then $A(c, x ! \wedge c)>0$.

The following Lemma shows the fundamental properties of $x$ ! which can be obtained from definition 3.7.

Lemma 3.11. Let $\mathrm{R}$ be a PSADFL. Then for any $a, b \in R$, we have the following:

1) $\quad A(a !, a \wedge a !)>0$ and $A(a, a \vee a !)>0$.

2) $A(0,0 !)>0$.

3) $\quad A(m, x \wedge m)>0$ if and only if $A(m, x !)>0$.

4) $\quad A\left(m, m_{1} !\right)>0$, for any maximal element $m_{1}$ of $R$.

5) If $b \in B_{A}(R)$, then $A(b \wedge m, b !)>0$.

6) $\quad A(x ! !, x !)>0$

7) $\quad A(x, y \wedge x)>0$, then $A(x !, y ! \wedge x !)>0$.

Theorem 3.12. Let R be a PSADFL. Then for any $a, b \in R$, we have the following:

1) $\quad A(a ! \wedge b !,(a \wedge b) !)>0$.

2) $\quad A((a \wedge b) !,(b \wedge a) !)>0$.

3) $\quad A(a ! \vee b !,(a \vee b) !)>0$.

4) $\quad A((a \vee b) !, b ! \vee a !)>0$.

Proof. 1. Let $a, \quad b \in R$. Then $A((a \wedge b) ! \wedge a ! \wedge b !, a ! \wedge b !)>0$. But $A(a ! \wedge b !,(a \wedge b) ! \wedge a ! \wedge b !)>0$.

Hence $A(a ! \wedge b !,(a \wedge b) !)>0$.

2) Let $a, b \in R$. Then $A((a \wedge b) !,(b \wedge a) !)=A(a ! \wedge b !,(b \wedge a) !)=A(b ! \wedge a !,(b \wedge a) !)=A((b \wedge a) !,(b \wedge a) !)=1>0$.

Hence $A((a \wedge b) !,(b \wedge a) !)>0$.

The proof of (3) and (4) follows on the same manner.

Theorem 3.13. $(R, A)$ is a PSADFL if and only if $[0, m]$ is a pseudo -supplemented fuzzy lattice.

Proof. Suppose $(R, A)$ is a pseudo-supplemented almost distributive fuzzy lattice. Let $x \in[0, m]$. Then 
$x ! \in B_{A}([0, M])$ and $A(x !, x \wedge x !)>0$.

Let $c \in B_{A}([0, m])$ and $A(c, x \wedge c)>0$. Then $A(c, x ! \wedge c)>0$.

Therefore $[0, m]$ is pseudo- supplemented fuzzy lattice.

Conversely, suppose $[0, m]$ is a pseudo-supplemented fuzzy lattice.

For $x \in R$ define $A\left(x !,(x \wedge m)^{*}=1\right.$, where $(x \wedge m)^{*}$ is a pseudo- Supplement of $x \wedge m \in[0, m]$. Then

$$
\begin{aligned}
A(x \wedge x !, x !)=A\left(x \wedge(x \wedge m)^{*}, x !\right) \\
\quad=A\left((x \wedge m) \wedge(x \wedge m)^{*}, x !\right) \\
=A\left((x \wedge m)^{*}, x !\right)=A(x !, x !)=1>0 .
\end{aligned}
$$

Let $b \in B_{A}(R)$ such that $A(b, x \wedge b)>0$. Then $b \wedge m \in B_{A}([0, m])$ and $A\left(b \wedge m,(x \wedge m)^{*}\right)>0$.

So that $A(x ! \wedge b \wedge m, b \wedge m)=A\left((x \wedge m)^{*} \wedge b \wedge m, b \wedge m\right)=A(b \wedge m, b \wedge m)=1>0$

Hence $A(b, x ! \wedge b)>0$. Thus $(R, A)$ is a PSADFL.

Theorem 3.14. $(R, A)$ is a PSADFL if and only if $P I(R)$ is a pseudo-supplemented fuzzy lattice.

\section{Discussion}

In this paper, we introduce the new concepts of Pseudo Supplemented Almost Distributive fuzzy Lattice as extension of Pseudo Supplemented Almost Distributive Lattice and this will lead for future development of the theory of algebra and its application.

\section{Conclusion}

The concept of pseudo-supplemented almost distributive fuzzy lattice is introduced. The idea of Birkhoff center in pseudo-supplemented almost distributive fuzzy lattice, the equivalence of pseudo-supplemented almost distributive lattice, and pseudo-supplemented almost distributive fuzzy lattice is investigated. Different characterizations of theorems, lemmas and propositions are proved.

\section{Acknowledgements}

The author of this paper expresses great thanks to the referee for their genuine comments.

\section{References}

[1] U. M. Swamy and G. C. Rao. (1981). Almost Distributive Lattice. J. Aust. Math. Soc (Sec A), 31(1981), pp. 77-91.

[2] G. C. Rao and Mihret Alemneh. (2015). Properties of Stone Almost Distributive Lattices, Asian-European Journal of Mathematics, Vol. 8, No. 1(2015), Doi: 101143-3557115300114.

[3] U. M. Swamy, G. C. Rao, and G. Nanaji. (2000). Pseudo-Complementation on Almost Distributive Lattice, South east Asian Bull. Math. 24(2000), pp. 95-104.

[4] G. C. Rao and Naveen Kakumanu. (2013). Pseudo-Supplemented Almost Distributive Lattices, South east Asian Bulletin of Mathematics (2013), Vol. 37, pp. 131-138.

[5] L. A. Zadeh. (1965). Fuzzy sets, inform and control 8(1965), pp. 338-353.

[6] Inheung Chon. (2016). Some properties of Fuzzy Lattices as Fuzzy Relatioms. Korean J. Math., 24(2016), No. 3, pp. 525-535.

[7] Berhanu, A., Yohannes, G., and Bekalu T. (2017). International Journal of Mathematics and its Applications, Vol. 5, Isue 1-C(2017), pp. 307-316.

[8] Berhanu, A. and Gerima, T. (2017). Birkhoff center of an Almost Distributive Fuzzy Lattice, International Journal of Computing Science and Applied Mathematics, Vol. 3, No. 2, August 2017, pp. 65-70.

[9] Berhanu, A., Mihret, A., and Gerima, T. (2018). B-Almost Distributive Fuzzy Lattice, Bulletin of the section of Logic, Vol. 47, 3(2018), pp. 171-185. http://dx.doi.org/10.18778/0138-0660.47.3.03. 